\title{
Mucosal breaks show same circumferential distribution in majority of patients with recurrent reflux esophagitis
}

\section{(ㅇ) $\circledast \Theta$}

\author{
Authors \\ Naoki Fukuda ${ }^{1,2}$, Norihisa Ishimura ${ }^{1}$, Mayumi Okada', Daisuke \\ Izumi ${ }^{1}$, Hironobu Mikami ${ }^{1}$, Eiko Okimoto ${ }^{1}$, Masahito Aimi ${ }^{1}$, Tsuyoshi \\ Mishiro $^{1}$, Naoki Oshima ${ }^{1}$, Shunji Ishihara ${ }^{1}$, Yoshikazu Kinoshita ${ }^{1}$

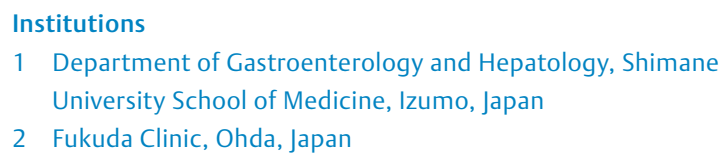

\begin{abstract}
Background and study aims Esophageal mucosal breaks in patients with reflux esophagitis (RE) have a unique circumferential distribution. However, the specific location of mucosal breaks during recurrence of RE remains unclear. We investigated the circumferential distribution of mucosal breaks in patients with recurrent RE and compared their location to that noted at the initial diagnosis.

Patients and methods We retrospectively enrolled patients with recurrent RE with Los Angeles (LA) grade A-C who were treated at our University Hospital between July 1996 and June 2014. The circumferential distribution of esophageal mucosal breaks was evaluated at the time of the initial diagnosis and again at the time of recurrence. Information regarding clinical parameters, including proton pump inhibitor administration, presence of hiatal hernia, and mucosal atrophy, was also reviewed.

Results A total of 114 patients with recurrent RE were examined during the study period, with a mean duration to recurrence after initial diagnosis of 39.4 months. The majority $(72.8 \%)$ had the same LA grade at recurrence. In addition, recurrent mucosal breaks in $96(84.2 \%)$ patients were observed to have occurred in the same circumferential location as at the initial diagnosis, while those in 18 $(15.8 \%)$ were observed in a different location. When recurrent lesions had a different location, the LA grade also tended to be different $(P=0.02)$.

Conclusions We found that most patients with recurrent RE developed lesions in the same circumferential location as noted for the initial lesions. Those in different locations at recurrence were associated with a change in LA grade.
\end{abstract}

\section{Introduction}

The prevalence of gastroesophageal reflux disease (GERD) has been increasing, especially in Western countries [1-3]. Although the reported prevalence is lower in Asian populations, GERD has become more prevalent in recent decades due to several factors, including a decrease in the rate of Helicobacter pylori (H. pylori) infection, westernization of eating habits, and increased obesity rate [4-7]. Patients with GERD suffer from heartburn and regurgitation, which are caused by reflux of stomach contents, leading to reductions in health-related quality of life and work productivity [8,9]. GERD patients will usually experience relapse once the prescribed drug is discontinued, because contributing factors, such as hiatal hernia, lower esophageal sphincter (LES) hypotension, and delayed gastric emptying are not corrected by acid inhibition. In addition, GERD is a risk factor for development of Barrett's esophagus and esophageal adenocarcinoma $[10,11]$, thus longitudinal management of affected patients is considered to be impor- tant. However, the long-term outcome of GERD patients with or without medical management remains to be fully clarified.

GERD can be classified into non-erosive reflux disease (NERD) and erosive reflux disease, or reflux esophagitis (RE), in which mucosal breaks are observed by endoscopy [12]. Severity in patients with the latter condition is associated with esophageal acid exposure time [13]. Previously, we focused on the circumferential distribution of esophageal mucosal breaks in patients with various grades of RE and found a unique asymmetrical circumferential distribution [14]. In that study, esophageal mucosal breaks in low-grade esophagitis [Los Angeles (LA) grade $A$ and $B$ ] cases [15] were mainly found on the right anterior wall of the distal esophagus. In contrast, those in cases of high-grade esophagitis (LA grade C) were mainly found on the posterior wall of the esophagus, suggesting a difference in acid reflux pattern between low- and high-grade RE in association with the circumferential distribution of mucosal breaks [13]. Given RE progresses to more severe forms over time, circumferential location of mucosal breaks may change at the time of re- 


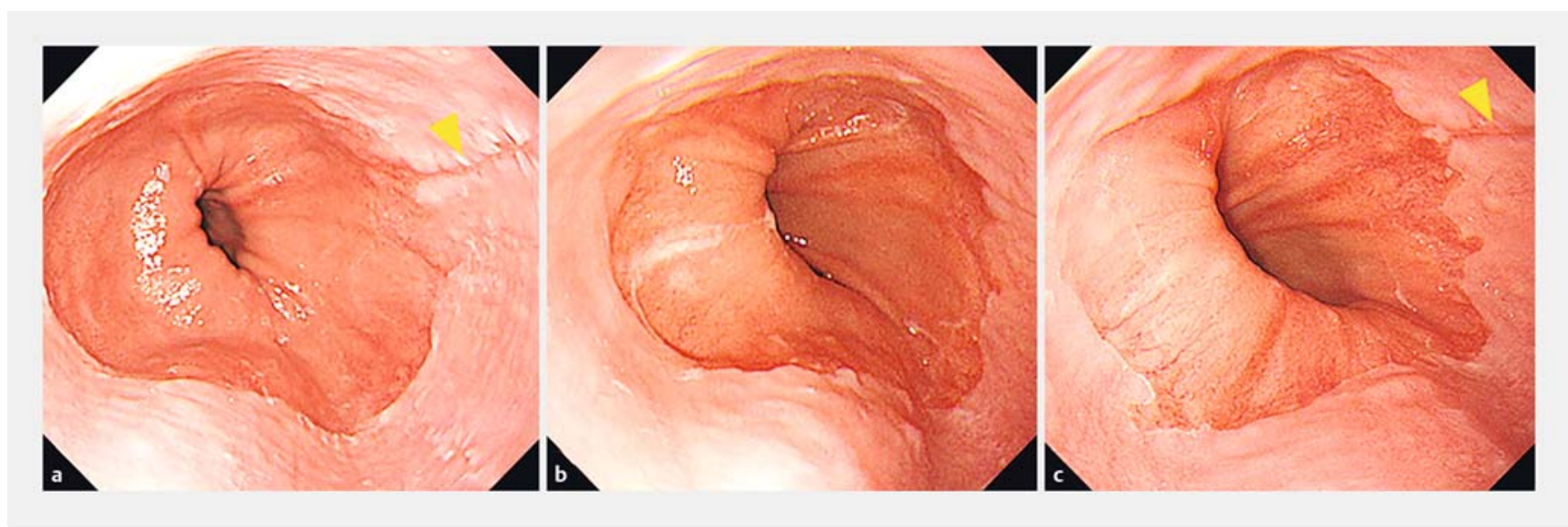

- Fig. 1 Circumferential distribution of esophageal mucosal breaks in a patient with recurrent RE. Shown are representative endoscopic findings of esophageal mucosal breaks in the same location at recurrence. a Esophageal mucosal break (arrowhead) observed at 2 o'clock at initial diagnosis. b Following PPI treatment, healing was confirmed. c At recurrence, a new mucosal break (arrowhead) was again observed at 2 o'clock, which was defined as recurrence at the same location as noted at the time of the initial diagnosis.

currence as compared to that at the time of initial diagnosis. However, it remains unclear whether the circumferential location of esophageal mucosal breaks changes during a long-term course of RE.

The aim of the current study was to investigate the circumferential distribution of mucosal breaks in patients with recurrent RE and compare to the location noted at the initial diagnosis.

\section{Patients and methods}

\section{Subjects}

We retrospectively reviewed the medical records of patients diagnosed with recurrent RE with LA grade A-C [15] at Shimane University Hospital between July 1996 and June 2014. All were initially given proton pump inhibitor (PPI) treatment for at least 2 months and healing of initial mucosal breaks (LA grade $\mathrm{N}$ or M) was confirmed by esophagogastroduodenoscopy (EGD) findings. Each was followed on a long-term basis under routine care at the discretion of the attending physician. Yearly followup EGD examinations were performed for screening of gastric cancer in some cases, and repeated EGD was also performed when symptom relapse occurred. Patients with new esophageal mucosal breaks were diagnosed as recurrent RE, irrespective of acid suppressive treatment, and enrolled in this study.

Endoscopic images with reports were separately reviewed by 3 of the authors (N.F, H.M, N.I). The examiners were blinded in regard to the clinical diagnosis of each case and compared each report with related endoscopic images to confirm presence and location of each lesion described by examining endoscopists. Endoscopic diagnosis was established by consensus of at least 2 of the 3. The grade and location of new mucosal breaks were evaluated to determine the circumferential distribution of the lesions, and their location was also compared to that at the time of the initial diagnosis. Information regarding clinical parameters, including demographics (age, sex), PPI or $\mathrm{H}_{2}$ receptor antagonist $\left(\mathrm{H}_{2} \mathrm{RA}\right)$ administration at the time of re- currence, presence of hiatal hernia, and gastric mucosal atrophy, was reviewed. A hiatal hernia was diagnosed when its length was greater than $2 \mathrm{~cm}$. The degree of gastric mucosal atrophy was endoscopically graded according to the Kimura-Takemoto classification. A grade of C1 - 3 for closed type indicated milder gastric mucosal atrophy, while a grade of $01-3$ for open type indicated more severe gastric mucosal atrophy. Patients with a history of upper gastrointestinal surgery were excluded. Patients who had taken any PPI or $\mathrm{H}_{2} \mathrm{RA}$ within 4 weeks prior to the initial diagnosis were also excluded. The protocol was approved by the ethics committee of Shimane University School of Medicine and the study was carried out in accordance with the principles of the Helsinki Declaration.

\section{Assessment of circumferential distribution of mucosal breaks}

The circumferential locations of mucosal breaks on the esophageal wall were evaluated as on a clock face, as follows. With the anterior wall of the esophagus always positioned at 0 o'clock, the 3 o'clock position was defined as the right lateral wall of the esophagus aligned with the lesser curvature of the stomach. Examining endoscopists described a clock face orientation of the esophageal lumen when they found the mucosal breaks in the endoscopic report. Patients with insufficient endoscopic reports and those with mucosal breaks whose location could not be clearly shown by endoscopic imaging were excluded. Subjects with recurrent RE were divided into 2 groups; recurrence at the same location and that at a different location. If mucosal breaks were observed at more than 1 location at the time of recurrence, those with at least 1 at the same location were defined as having lesions at the same location.

Representative endoscopic findings in a patient with mucosal breaks at the same locations are shown in - Fig. 1, while those in a patient with mucosal breaks in a different location

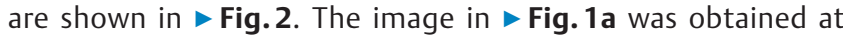
the time of the initial diagnosis and shows a mucosal break (arrowhead) at the 2 o'clock location. Following PPI treatment, 

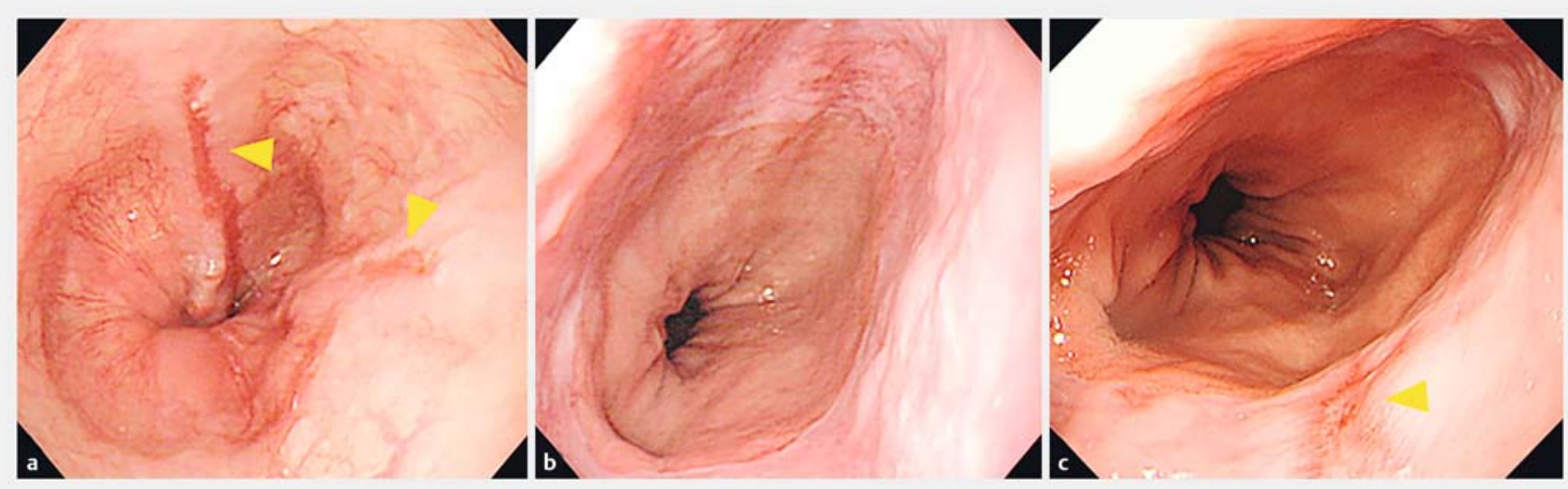

- Fig. 2 Circumferential distribution of esophageal mucosal breaks in a patient with recurrent RE. Shown are representative endoscopic findings of esophageal mucosal breaks at a different location at recurrence. a Esophageal mucosal break (arrowheads) was observed at 0 , and 3 o'clock at the initial diagnosis. B Following PPI treatment, healing was confirmed. c At recurrence, a new mucosal break (arrowhead) was observed at 6 o'clock with severe hiatal hernia, which was defined as recurrence at a different location as compared to that noted at the time of initial diagnosis.

- Table 1 Clinical characteristics of patients with recurrent reflux esophagitis.

\begin{tabular}{|l|l|}
\hline Number of patients & 114 \\
\hline Male, no. (\%) & $76(67)$ \\
\hline $\begin{array}{l}\text { Age at initial diagnosis, years, } \\
\text { mean } \pm \text { SD (range) }\end{array}$ & $65.5 \pm 12.5(34-86)$ \\
\hline $\begin{array}{l}\text { Age at recurrence, years, } \\
\text { mean } \pm \text { SD (range) }\end{array}$ & $68.7 \pm 12.2(42-89)$ \\
\hline $\begin{array}{l}\text { Time to recurrence, months, } \\
\text { mean } \pm \text { SD (range) }\end{array}$ & $39.4 \pm 31.4(3-142)$ \\
\hline $\begin{array}{l}\text { LA grade at initial diagnosis, no. } \\
\text { (A/B/C) }\end{array}$ & $71 / 34 / 9$ \\
\hline $\begin{array}{l}\text { LA grade at recurrence, no. (A/B/C) } \\
\text { PPI administration at recurrence, } \\
\text { no. (\%) }\end{array}$ & $68 / 38 / 8$ \\
\hline $\begin{array}{l}\mathbf{H}_{2} \mathrm{RA} \text { administration at recurrence, } \\
\text { no. (\%) }\end{array}$ & $10(39)$ \\
\hline \begin{tabular}{l} 
Hiatal hernia, no. (\%) \\
\hline Atrophic gastritis (open type), no. (\%)
\end{tabular} & $23(20)$ \\
\hline $\begin{array}{l}\text { PPI, proton pump inhibitor; } \mathrm{H}_{2} \mathrm{RA}, \text { histamine } \mathrm{H}_{2} \text { receptor antagonist; LA, Los } \\
\text { Angeles classification of gastroesophageal reflux disease }\end{array}$ \\
\hline
\end{tabular}

healing of the mucosal break seen at the initial diagnosis was confirmed ( $\triangleright$ Fig. 1b). At the time of recurrence, a new mucosal break was observed in the same location (2 o'clock) as the initial lesion ( $\triangleright$ Fig. 1c). $\$$ Fig. 2 a presents an image of mucosal breaks (arrowhead) located at 0 , and 3 o'clock that were observed at the initial diagnosis. Following PPI treatment, healing of the mucosal breaks seen at the initial diagnosis was confirmed ( $\triangleright$ Fig. 2b). At recurrence, a new mucosal break was observed at position corresponding to 6 o'clock with severe hiatal hernia ( $\triangleright$ Fig. $2 c$ ), indicating recurrent mucosal break at different location.

Background factors, including sex, age at initial diagnosis, age at recurrence, time to recurrence, LA grade at the initial diagnosis and at recurrence, administration of PPI or $\mathrm{H}_{2} \mathrm{RA}$ at recurrence, and the presence of hiatal hernia and atrophic gastritis were compared between cases with recurrent lesions at the same location and those with such recurrence at different locations.

\section{Statistical analysis}

Statistical analyses were performed using chi-squared and Mann - Whitney U-tests. After identification of significant predictors by univariate analysis, logistic regression analysis was performed to calculate the odds ratio with confidence interval (CI) for independent predictors of a different location of mucosal breaks at recurrence. A value of $P<0.05$ was considered to indicate a statistically significant difference. All statistical analyses were performed using the SPSS statistical analysis software package (version 22.0 for the PC, Chicago, IL, USA).

\section{Results}

\section{Patient profiles}

The clinical characteristics of patients with recurrent reflux esophagitis are shown in $>$ Table 1 . We enrolled 114 patients (76 males, 38 females) whose mean $( \pm$ SD) age was $65.5 \pm 12.5$ years (range 34-86 years) at the time of the initial diagnosis. The number of cases with LA grade $A$ was 71 , while 34 were LA grade $B$ and 9 were LA grade $C$, while those numbers at recurrence were 68,38 , and 8 , respectively. The mean time to recurrence after the initial diagnosis was $39.4 \pm 31.4$ months (range 3-142 months). PPIs were administrated to 44 patients (37 with regular or double dose, 7 with half dose), while $\mathrm{H}_{2}$ receptor antagonists were administered to 10 patients at the time of recurrence. 
- Table 2 Change in severity of LA grade at recurrence.

LA classification at initial diagnosis

\begin{tabular}{|l|l|l|l|}
\hline & $\begin{array}{l}\text { LA-A } \\
(n=68)\end{array}$ & $\begin{array}{l}\text { LA-B } \\
(n=38)\end{array}$ & $\begin{array}{l}\text { LA-C } \\
(n=8)\end{array}$ \\
\hline LA-A $(n=71)$ & 56 & 14 & 1 \\
& $(78.9 \%)$ & $(19.7 \%)$ & $(1.4 \%)$ \\
\hline LA-B $(n=34)$ & 10 & 22 & 2 \\
& $(29.4 \%)$ & $(64.7 \%)$ & $(5.9 \%)$ \\
\hline LA-C $(n=9)$ & 2 & 2 & 5 \\
\hline & $(22.2 \%)$ & $(22.2 \%)$ & $(55.6 \%)$ \\
\hline
\end{tabular}

LA, Los Angeles classification of gastroesophageal reflux disease

\section{Change in severity of LA grade at recurrence}

First, we evaluated the change in severity of LA grade at recurrence ( $\triangleright$ Table 2$)$. Fifty-six $(78.9 \%)$ of our RE patients with LA grade $A$ had recurrence with the same grade, while 15 (21.1\%) progressed to a more severe form (14 grade $B, 1$ grade $C)$. Likewise, 22 (64.7\%) with LA grade B had the same grade at recurrence, while only 2 (5.9\%) progressed to grade C. In contrast, 10 (29.4\%) had a milder form of RE (grade A) at recurrence. Taken together, only 17 (14.9\%) progressed to a more severe grade of RE, while the majority $(72.8 \%)$ had recurrence at the same LA grade as at the time of the initial diagnosis. When the patients were divided into those with PPI administration at recurrence $(n=44)$ or those without administration at recurrence $(n=70)$, the majority had the same LA grade at recurrence in both groups (63.6\% of subjects with PPI administration, $78.6 \%$ of subjects without PPI), while the ratio of patients with improvement to a milder grade was higher in those with PPI administration than in those without PPI administration $(20.5 \%$ of subjects with PPI administration, $7.1 \%$ of subjects without PPI, $\mathrm{p}<0.05)$.

\section{Circumferential distribution of mucosal breaks}

Next, we assessed the circumferential distribution of mucosal breaks in the esophageal wall in our patients at the time of initial diagnosis and at recurrence of RE. That distribution at the initial diagnosis is shown in $\mathbf{F i g . 3}$. In patients with LA grade $A$, mucosal breaks were frequently located at the 2 o'clock position, while those in patients with grade B were located at positions from 2 to 3 o'clock. In contrast, in patients with grade $C$, the mucosal breaks were mainly located on the posterior wall of the esophagus. These findings were consistent with those obtained in our previous study [14]. As shown in > Fig. 4, the circumferential distribution of mucosal breaks at recurrence was similar to the pattern seen at the initial diagnosis, suggesting that it was consistent and closely associated with RE severity. When that distribution were compared between those with PPI $(n=44)$ and without PPI at recurrence $(n=70)$, the pattern of that distribution at recurrence was not different between the groups (data not shown), suggesting that PPI administration was not strongly associated with the circumferential distribution of mucosal breaks.

\section{Comparison of circumferential location of mucosal breaks at initial diagnosis and recurrence}

New esophageal mucosal breaks in the same location as the initial lesions ( $\triangleright$ Fig. 1 ) were found in 96 patients (84.2\%), while those in a different location ( $\triangleright$ Fig. 2 ) were found in 18 (15.8 $\%)$, indicating that the majority had recurrence at the same location. To evaluate the relationship between mucosal break location and change in severity of LA grade at recurrence, we divided the patients into 3 groups based on findings at recurrence; same LA grade as at initial diagnosis $(n=83)$, progression to more severe grade $(n=17)$, and improvement to milder grade $(n=14)$ ( $\boldsymbol{F}$ Fig. 5$)$. Interestingly, in most of the patients $(89.2 \%)$ with the same severity as seen at the initial diagnosis, mucosal breaks were observed in the same location as at the initial diagnosis, while those with either progression or improvement in severity had lesions in the same location as at the initial diagnosis less frequently $(29.4$, and $28.6 \%$, respectively $P<$ $0.05)$. At recurrence, PPIs were more frequently administered to patients with improvement to a milder grade as compared to the others $(64.3 \%$ of patients with milder grade, $33.7 \%$ of patients with same grade, $41.2 \%$ of patients with progression to more severe grade; $P<0.05)$.

We also compared clinical parameters between patients with recurrent lesions in the same location as the initial lesions $(n=96)$ and patients with those in different locations $(n=18)$ ( $\triangleright$ Table 3). There were no significant differences in regard to age, time period to recurrence, administration of PPIs at recurrence, or presence of hiatal hernia and atrophic gastritis between the groups. However, recurrence with a different LA grade severity was significantly associated with a different location of mucosal breaks at recurrence $(P<0.05)$. Logistic regression analysis findings confirmed that a change in severity of lesions was a positive independent factor for different location of recurrent mucosal breaks (OR 6.07, 95\% Cl 1.73-21.28, $P<$ $0.01)$.

\section{Discussion}

Patients with RE, easily recognized based on EGD findings, often develop complications, such as bleeding, deep ulceration, strictures, Barrett's esophagus, and esophageal adenocarcinoma $[10,11]$. However, there is scant information available regarding their long-term clinical course or type of recurrence. In the present study, RE patients were found to usually experience recurrence at the same LA grade, as most did not progress to a more severe grade during the follow-up observations.

Prevalence of GERD has been increasing in Asian populations in recent decades, mainly due to decreases in rates of $\mathrm{H}$. pylori infection [4] and increases in obesity $[6,7,16]$, though the rate of obesity (body mass index $\geq 30 \mathrm{~kg} / \mathrm{m}^{2}$ ) is much lower in Asian as compared to Western countries $[17,18]$. Gastric acid secretion is another important factor for development of GERD. Studies conducted in the $1990 \mathrm{~s}$ of Japanese subjects showed that gastric acid secretion levels increased from the $1970 \mathrm{~s}$ to 1990 s, though they remained lower as compared to Europeans and North Americans [19-21]. In addition, those levels have 


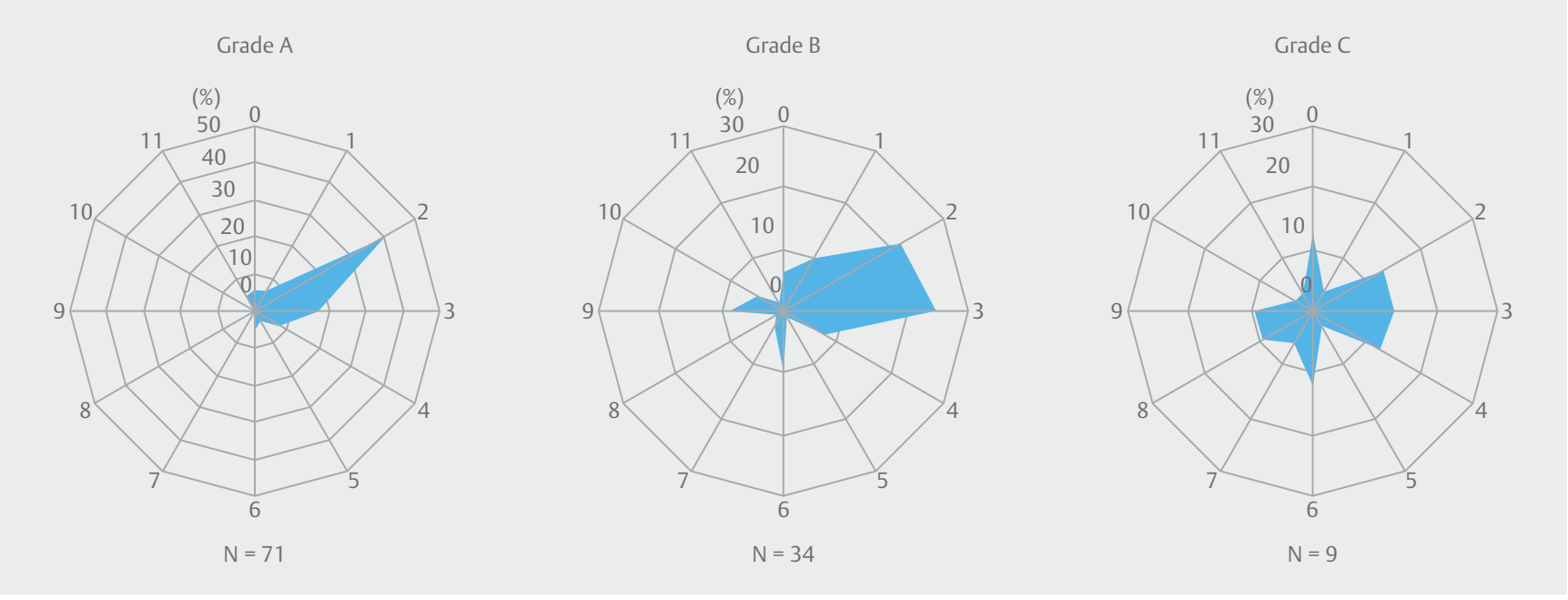

- Fig. 3 Circumferential distribution of esophageal mucosal breaks in patients with RE and LA grade of A, B, or C (image A, B, C, respectively) at the time of the initial diagnosis. Locations are shown in terms of clock face orientation.

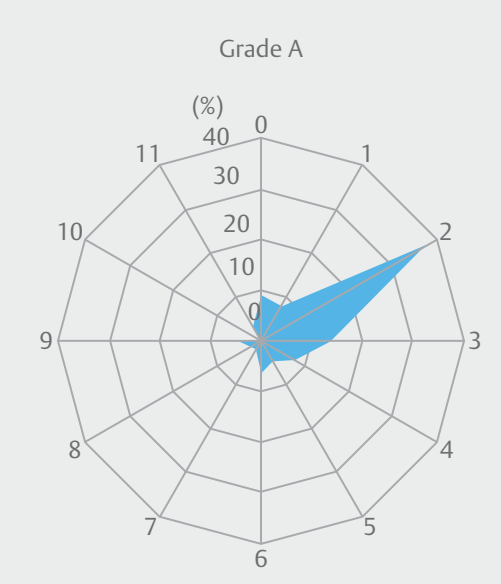

$N=68$

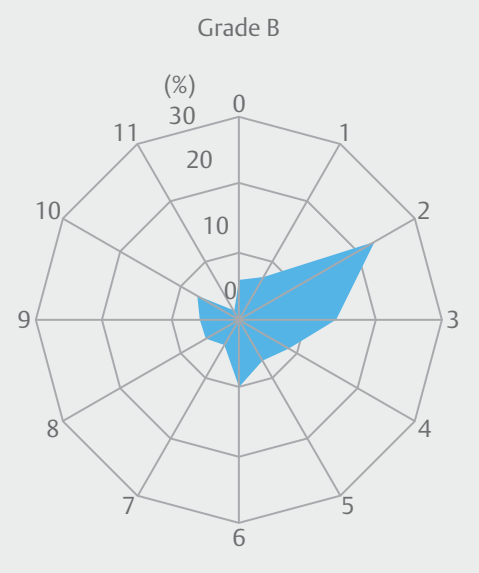

$N=38$

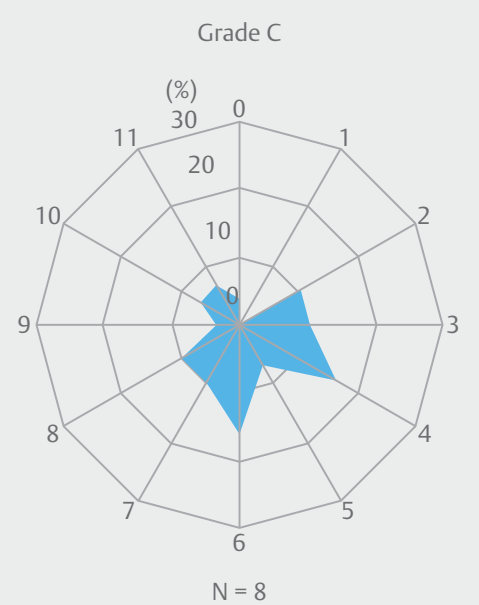

- Fig. 4 Circumferential distribution of esophageal mucosal breaks in patients with RE and an LA grade of A, B, or C (image A, B, C, respectively) at the time of recurrence. Locations are shown in terms of clock face orientation. The location was similar to that noted at the time of initial diagnosis in each patient.

not increased over the past 2 decades in Japanese [22, 23]. These factors may be related to the lower prevalence of GERD in Asian individuals as compared to Western populations. Consistent with the present results, approximately $90 \%$ of RE cases in Japan have been found to be low grade, such as LA grade A or $B$ [3].

As for the natural history of GERD, Manabe et al. followed 105 patients with LA grade A or B RE who did not receive medical treatment for that condition for a mean 5.5 years (range $2.0-8.8$ years) [24]. In that study, only $10.5 \%$ of the patients progressed to a more severe grade (mostly grade C) and none progressed to Barrett's esophagus. They also evaluated factors associated with development of severe esophagitis. Risk factors for progressive disease were found to be increased age, female sex, presence of symptoms at initial diagnosis, presence of hia- tal hernia, no atrophic gastritis, and no $H$. pylori infection. Our results were consistent with theirs and suggest little progression in severity over time regardless of grade. Furthermore, findings from a large multicenter study conducted in Europe of 2721 patients who completed the 5-year follow-up protocol suggest that most GERD patients remain stable or show improvement when receiving current routine clinical care [25]. Taken together, in most patients, low-grade RE does not progress over time, though factors predictive of progression to more severe esophagitis must be clarified in order to identify those at risk.

Consistent with our previous findings [14], the current study showed that the circumferential distribution of esophageal mucosal breaks varies based on LA grade showing the severity of esophagitis, regardless of the timing of the endoscopic obser- 

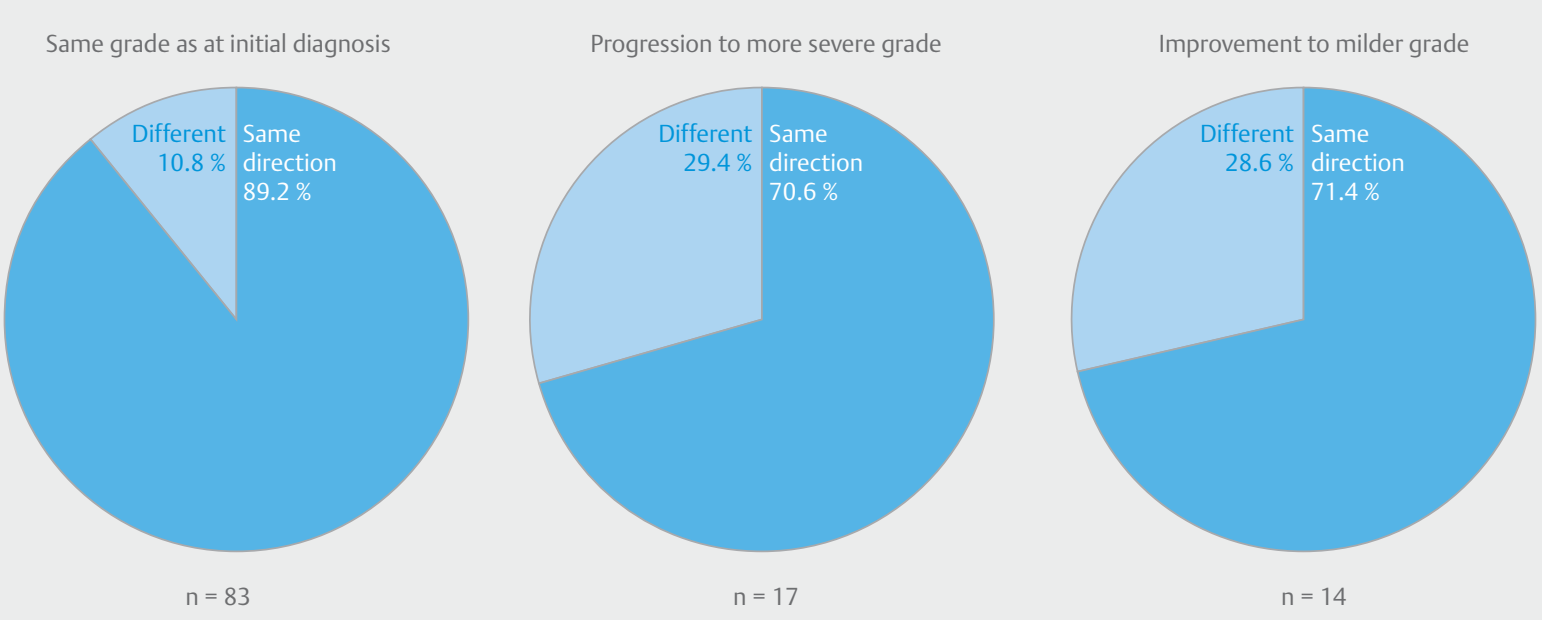

- Fig. 5 Relationship between location of mucosal break and change in severity of LA grade at recurrence. Patients were divided into three groups; same LA grade as at initial diagnosis $(n=83)$, progression to more severe grade $(n=17)$, and improvement to milder grade $(n=14)$. Most of the patients $(89.2 \%$ ) with the same grade at the time of recurrence showed mucosal breaks in the same location as noted at the initial diagnosis, while those with progression or improvement of mucosal breaks at recurrence less frequently had lesions in the same location as noted at the initial diagnosis (29.4, and 28.6, respectively).

- Table 3 Characteristics of patients with recurrent RE in same and different locations.

\begin{tabular}{|c|c|c|c|}
\hline & $\begin{array}{l}\text { Same } \\
(n=96)\end{array}$ & $\begin{array}{l}\text { Different } \\
(n=18)\end{array}$ & $P$ value \\
\hline Male, no. (\%) & $67(70)$ & $9(50)$ & 0.10 \\
\hline Age at initial diagnosis, years, mean \pm SD & $64.8 \pm 13.1$ & $69.2 \pm 8.8$ & 0.25 \\
\hline Age at recurrence, years, mean \pm SD & $68.0 \pm 12.8$ & $72.5 \pm 8.0$ & 0.23 \\
\hline Time to recurrence, months, mean \pm SD & $39.4 \pm 30.4$ & $39.5 \pm 37.2$ & 0.68 \\
\hline LA grade at initial diagnosis, no. (A/B/C) & $59 / 28 / 9$ & $12 / 6 / 0$ & 0.20 \\
\hline Recurrence with different LA grade, no. (\%) & $22(23)$ & $9(50)$ & 0.02 \\
\hline PPI administration at recurrence, no. (\%) & $37(39)$ & $7(39)$ & 0.98 \\
\hline $\mathrm{H}_{2} \mathrm{RA}$ administration at recurrence, no. (\%) & $7(7)$ & $3(17)$ & 0.95 \\
\hline Hiatal hernia, no. (\%) & $75(78)$ & $13(72)$ & 0.58 \\
\hline Atrophic gastritis (open type), no. (\%) & $17(18)$ & $6(33)$ & 0.13 \\
\hline
\end{tabular}

vation (initial diagnosis or recurrence) and presence or absence of PPI administration. Esophageal mucosal breaks were mostly found on the right anterior wall (2 o'clock) of the esophagus in RE patients with LA grade of $A$ or $B$, while those were often seen on the posterior wall of the esophagus in patients with highgrade RE (grade C). Potential pathophysiologic explanations underlying the circumferential distribution of mucosal breaks in RE include the functional structure of the lower esophageal sphincter and nonuniform asymmetric distribution of esophageal acid exposure [26-28]. Interestingly, circumferential intraesophageal pressure at the LES has been reported to be asymmetrical [29], while a recent study that used 3-dimensional high-resolution manometric imaging revealed decreased competency within the right-side portion of the LES pressure zone as compared to the left side [30]. These findings suggest that asymmetrical LES pressure caused by anatomical proportion may be a major cause of asymmetrical gastroesophageal acid reflux and is correlated with the predominant circumferential location of mucosal breaks in the esophagus. Patients with grade $C$ or D esophagitis show decreased LES pressure with an impaired reflux barrier caused by the factors including hiatal hernia, thus they often have severe nocturnal gastroesophageal reflux when lying in bed [13]. Therefore, mucosal breaks are prone to be found on the posterior wall of the esophagus.

The current study is the first to show that most patients with recurrent $R E$ develop mucosal breaks at the same circumferen- 
tial location as noted at the time of initial diagnosis. When clinical parameters in recurrent cases were compared between those with the same and different locations of new lesions, recurrence with a changed LA severity grade, irrespective of improvement or progression, was the only significant parameter. Our results suggest that acid reflux mechanisms in patients with RE differ among the different grades and the pathogenetic mechanism does not usually change over the long term. When a new lesion is found in a different circumferential location, a different acid reflux mechanism, such as having hiatal hernia, should be suspected as predominant in that patient. Thus, the circumferential location of lesions observed during follow-up endoscopic examinations may be an important parameter to diagnose regarding disease progression.

Another importance of this study is that persistent higher level of gastric acid and/or bile acid reflux at the same circumferential location may result in mucosal breaks associated with Barrett's esophagus (BE), and BE-associated adenocarcinoma. We previously investigated the location of short segment $B E$ (SSBE) and early neoplastic lesion in patients with SSBE, and reported their predominant distribution on the right anterior wall of the esophagus [31]. Our finding was confirmed by subsequent several promising studies [32,33]. In addition, recent report by Omae et al. revealed that the location of superficial BEassociated adenocarcinoma mostly corresponds to the direction of maximal total duration of acid or non-acid reflux [34]. Because the prognosis of patients with advanced-stage BEassociated adenocarcinoma remains poor, early detection of neoplastic lesion in those with BE has led to recent interest in effective treatment. Our data suggest that precise inspection of this area is important both in patients with suspected $\mathrm{BE}$ and in patients undergoing their surveillance of BE.

There are several limitations associated with this study, including its retrospective nature and all patients being treated at the same tertiary referral center. Although the circumferential distribution of esophageal mucosal breaks was determined retrospectively based on recorded endoscopic images with reports, we focused on lesions in the area of the esophago-gastric junction (EGJ). We previously reported the clinical significance of asymmetrical distribution of lesions in the EG], including mucosal breaks in patients with RE, SSBE, esophageal adenocarcinoma, Mallory-Weiss tears, and raptured esophageal varices $[27,35]$. Therefore, we consider that our institution has adequate experience with and uses a standardized protocol for use of endoscopic photo series in our endoscopy database, and the results obtained in this study are thought to be reliable. Nevertheless, a prospective study is needed to clarify the timecourse changes in circumferential distribution of esophageal mucosal breaks in patients with RE.

\section{Conclusions}

In summary, we found that most of our patients with recurrent RE developed lesions in the same circumferential location as noted at the initial diagnosis. Those in different locations were associated with change of LA grade with recurrence.

\section{Competing interests}

None

References

[1] El-Serag HB, Sweet S, Winchester CC et al. Update on the epidemiology of gastro-oesophageal reflux disease: a systematic review. Gut 2014; 63: $871-880$

[2] Kinoshita $Y$, Adachi K, Hongo M et al. Systematic review of the epidemiology of gastroesophageal reflux disease in Japan. J Gastroenterol 2011; 46: 1092 - 1103

[3] Fujiwara Y, Arakawa T. Epidemiology and clinical characteristics of GERD in the Japanese population. J Gastroenterol 2009; 44: 518-534

[4] Kamada T, Haruma K, Ito M et al. Time Trends in Helicobacter pylori Infection and Atrophic Gastritis Over 40 Years in Japan. Helicobacter 2015; 20: $192-198$

[5] Matsuki N, Fujita T, Watanabe N et al. Lifestyle factors associated with gastroesophageal reflux disease in the Japanese population. J Gastroenterol 2013; 48: 340-349

[6] Niigaki M, Adachi K, Hirakawa K et al. Association between metabolic syndrome and prevalence of gastroesophageal reflux disease in a health screening facility in Japan. J Gastroenterol 2013; 48: 463-472

[7] Okimoto E, Ishimura N, Morito Y et al. Prevalence of gastroesophageal reflux disease in children, adults, and elderly in the same community. J Gastroenterol Hepatol 2015; 30: 1140-1146

[8] Suzuki H, Matsuzaki J, Masaoka T et al. Greater loss of productivity among Japanese workers with gastro-esophageal reflux disease (GERD) symptoms that persist vs resolve on medical therapy. Neurogastroenterol Motil 2014; 26: $764-771$

[9] Tack J, Becher A, Mulligan C et al. Systematic review: the burden of disruptive gastro-oesophageal reflux disease on health-related quality of life. Aliment Pharmacol Ther 2012; 35: 1257-1266

[10] Cook MB, Corley DA, Murray L] et al. Gastroesophageal reflux in relation to adenocarcinomas of the esophagus: a pooled analysis from the Barrett's and Esophageal Adenocarcinoma Consortium (BEACON). PLoS One 2014; 9: e103508

[11] Ronkainen J, Talley NJ, Storskrubb T et al. Erosive esophagitis is a risk factor for Barrett's esophagus: a community-based endoscopic follow-up study. Am J Gastroenterol 2011; 106: 1946 - 1952

[12] Vakil N, van Zanten SV, Kahrilas P et al. The Montreal definition and classification of gastroesophageal reflux disease: a global evidencebased consensus. Am J Gastroenterol 2006; 101: 1900 - 1920; quiz 1943

[13] Adachi K, Fujishiro H, Katsube T et al. Predominant nocturnal acid reflux in patients with Los Angeles grade $C$ and $D$ reflux esophagitis. J Gastroenterol Hepatol 2001; 16: 1191-1196

[14] Katsube T, Adachi K, Furuta K et al. Difference in localization of esophageal mucosal breaks among grades of esophagitis. J Gastroenterol Hepatol 2006; 21: 1656 - 1659

[15] Lundell LR, Dent J, Bennett JR et al. Endoscopic assessment of oesophagitis: clinical and functional correlates and further validation of the Los Angeles classification. Gut 1999; 45: 172-180

[16] Adachi K, Mishiro T, Tanaka S et al. Gender differences in the timecourse changes of reflux esophagitis in Japanese patients. Intern Med 2015; 54: 869-873

[17] Matsushita Y, Takahashi Y, Mizoue T et al. Overweight and obesity trends among Japanese adults: a 10-year follow-up of the JPHC Study. Int J Obes (Lond) 2008; 32: 1861 - 1867

[18] Ogden CL, Carroll MD, Kit BK et al. Prevalence of childhood and adult obesity in the United States, 2011-2012. Jama 2014; 311: 806-814 
[19] Feldman M, Richardson CT, Lam SK et al. Comparison of gastric acid secretion rates and serum pepsinogen I and II concentrations in Occidental and Oriental duodenal ulcer patients. Gastroenterology 1988; 95: 630-635

[20] Goldschmiedt M, Barnett CC, Schwarz BE et al. Effect of age on gastric acid secretion and serum gastrin concentrations in healthy men and women. Gastroenterology 1991; 101: 977 - 990

[21] Kinoshita Y, Kawanami C, Kishi K et al. Helicobacter pylori independent chronological change in gastric acid secretion in the Japanese. Gut 1997; 41: $452-458$

[22] Ishimura N, Owada Y, Aimi M et al. No increase in gastric acid secretion in healthy Japanese over the past two decades. J Gastroenterol 2015; 50: $844-852$

[23] lijima K, Koike T, Abe Y et al. Time series analysis of gastric acid secretion over a 20-year period in normal Japanese men. J Gastroenterol 2015; 50: $853-861$

[24] Manabe N, Yoshihara M, Sasaki A et al. Clinical characteristics and natural history of patients with low-grade reflux esophagitis. J Gastroenterol Hepatol 2002; 17: 949 - 954

[25] Malfertheiner P, Nocon M, Vieth $M$ et al. Evolution of gastro-oesophageal reflux disease over 5 years under routine medical care-the ProGERD study. Aliment Pharmacol Ther 2012; 35: 154-164

[26] Ishimura N, Okada M, Mikami H et al. Pathophysiology of Barrett's Esophagus-Associated Neoplasia: Circumferential Spatial Predilection. Digestion 2014; 89: 291 - 298

[27] Kinoshita Y, Furuta K, Adachi K et al. Asymmetrical circumferential distribution of esophagogastric junctional lesions: anatomical and physiological considerations. J Gastroenterol 2009; 44: 812-818
[28] Ohara S, Furuta K, Adachi K et al. Radially asymmetric gastroesophageal acid reflux in the distal esophagus: examinations with novel $\mathrm{pH}$ sensor catheter equipped with 8 pH sensors. J Gastroenterol 2012; 47: $1221-1227$

[29] Liu J, Parashar VK, Mittal RK. Asymmetry of lower esophageal sphincter pressure: is it related to the muscle thickness or its shape? Am J Physiol 1997; 272: G1509-1517

[30] Kwiatek MA, Pandolfino JE, Kahrilas PJ. 3D-high resolution manometry of the esophagogastric junction. Neurogastroenterol Motil 2011; 23: e461 - e469

[31] Moriyama N, Amano Y, Okita K et al. Localization of early-stage dysplastic Barrett's lesions in patients with short-segment Barrett's esophagus. Am J Gastroenterol 2006; 101: 2666-2667

[32] Bibbo S, laniro G, Ricci R et al. Barrett's oesophagus and associated dysplasia are not equally distributed within the esophageal circumference. Dig Liver Dis 2016; 48: 1043 - 1047

[33] Enestvedt BK, Lugo R, Guarner-Argente C et al. Location, location, location: does early cancer in Barrett's esophagus have a preference? Gastrointest Endosc 2013; 78: 462 -467

[34] Omae M, Fujisaki ], Shimizu T et al. Correlation of the location of superficial Barrett's esophageal adenocarcinoma (s-BEA) with the direction of gastroesophageal reflux. Endosc Int Open 2016; 4: E515E520

[35] Okada M, Ishimura N, Shimura S et al. Circumferential distribution and location of Mallory-Weiss tears: recent trends. Endosc Int Open 2015; 03: E418-E424 